\author{
Correspondence \\ Takuya Nihira \\ nihira@icb.osaka-u.ac.jp
}

Received 5 April 2011

Revised 1 August 2011

Accepted 3 August 2011

\section{VisG is essential for biosynthesis of virginiamycin $S$, a streptogramin type B antibiotic, as a provider of the nonproteinogenic amino acid phenylglycine}

\author{
Fitria Ningsih, ${ }^{1}$ Shigeru Kitani, ${ }^{1}$ Eriko Fukushima ${ }^{1}$ and Takuya Nihira ${ }^{1,2}$ \\ ${ }^{1}$ International Center for Biotechnology, Osaka University, 2-1, Yamadaoka, Suita, \\ Osaka 565-0871, Japan \\ ${ }^{2}$ MU-OU Collaborative Research Center for Bioscience and Biotechnology, Faculty of Science, \\ Mahidol University, Rama VI Rd, Bangkok 10400, Thailand
}

\begin{abstract}
A streptogramin type B antibiotic, virginiamycin S (VS), is produced by Streptomyces virginiae, together with a streptogramin type $A$ antibiotic, virginiamycin $M_{1}(V M)$, as its synergistic counterpart. VS is a cyclic hexadepsipeptide containing a nonproteinogenic amino acid, Lphenylglycine (L-pheGly), in its core structure. We have identified, in the left-hand extremity of the virginiamycin supercluster, two genes that direct VS biosynthesis with L-pheGly incorporation. Transcriptional analysis revealed that vis $F$, encoding a nonribosomal peptide synthetase, and vis $G$, encoding a protein with homology to a hydroxyphenylacetyl-CoA dioxygenase, are under the transcriptional regulation of virginiae butanolide (VB), a small diffusing signalling molecule that governs virginiamycin production. Gene deletion of visG resulted in complete loss of VS production without any changes in VM production, suggesting that visG is required for VS biosynthesis. The abolished VS production in the visG disruptant was fully recovered either by the external addition of pheGly or by gene complementation, which indicates that VisG is involved in VS biosynthesis as the provider of an L-pheGly molecule. A feeding experiment with L-pheGly analogues suggested that VisF, which is responsible for the last condensation step, has high substrate specificity toward L-pheGly.
\end{abstract}

\section{INTRODUCTION}

Virginiamycin S (VS) (Fig. 1a) is a cyclic hexadepsipeptide antibiotic that belongs to the type B compounds in the streptogramin family. In Streptomyces virginiae, VS is coproduced with a polyunsaturated macrolactone antibiotic, virginiamycin $M_{1}(V M)$, which belongs to the type A compounds in the streptogramin family. Both antibiotics show strong synergistic bactericidal activity against a wide range of Gram-positive bacteria (Di Giambattista et al., 1989). Although virginiamycin has been used widely as a performance promoter in animal husbandry, human application of virginiamycin has been limited by its poor water solubility. However, Barrière et al. (1998) succeeded

Abbreviations: A, adenylation; D-amBu, D- $\alpha$-aminobutyric acid; $\mathrm{BCDH}$, branched-chain $\alpha$-keto acid dehydrogenase; C, condensation; Hpg, p-hydroxyphenylglycine; $\mathrm{M}$, methylation; NRPS, nonribosomal peptide synthetase; PCP, peptidyl carrier protein; L-pheGly, L-phenylglycine; qRT-PCR, quantitative RT-PCR; VB, virginiae butanolide; TE, thioesterase; VM, virginiamycin $M_{1}$; VS, virginiamycin $S$.

The DDBJ/EMBL/GenBank accession number for the sequence reported in this paper is AB621357.

A supplementary table, listing oligonucleotide primers used in this study, is available with the online version of this paper. in creating a Streptomyces pristinaespiralis mutant that produces derivatives of pristinamycin I, another type B compound in the streptogramin family, which show greater biological activity and higher water solubility than their natural counterparts. Furthermore, chemical modification of virginiamycin has led to the design of watersoluble derivatives, such as quinupristin and dalfopristin, which are both in use as therapeutic drugs (e.g. Synercid) against vancomycin-resistant Enterococcus faecium and other Gram-positive bacteria (Manzella, 2001). Thus, the importance of the streptogramin family, including virginiamycin, has increased dramatically.

VS is synthesized by stepwise condensation of two proteinogenic and five nonproteinogenic amino acids: 3hydroxypicolinic acid (derived from L-lysine) as a starter, followed by incorporation of $\mathrm{L}$-threonine, $\mathrm{D}-\alpha$-aminobutyric acid (D-amBu), L-proline, $N$-methyl-L-phenylalanine, 4-oxoL-pipecolic acid (derived from L-lysine) and L-phenylglycine (L-pheGly) (Fig. 1a) (Yamada et al., 1997). It has been postulated that nonribosomal peptide synthetase (NRPS) is involved in VS biosynthesis. Previously, we cloned plausible NRPS genes (visE and truncated visF) necessary for assembling the VS framework, which are located in the region downstream of $v m s T$ (a pathway-specific regulatory 
(a)

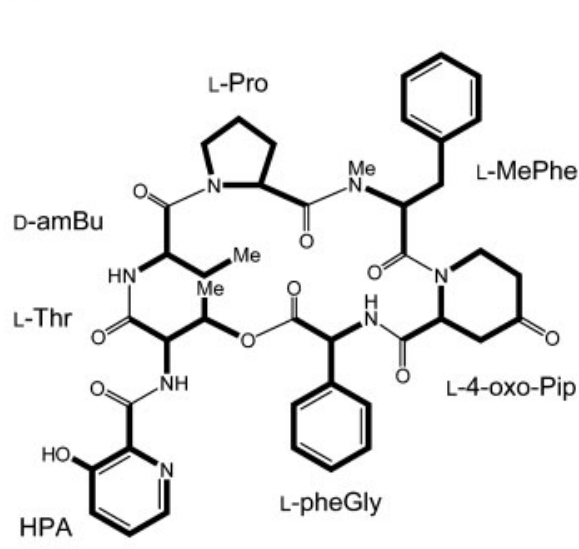

(b)

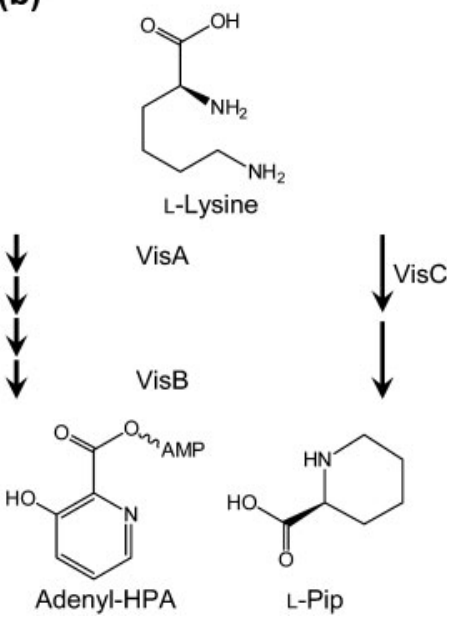

Fig. 1. Chemical structure of virginiamycin $S$ from $S$. virginiae and schematic representation of adenyl-HPA (hydroxypicolinic acid) and LPip (pipecolic acid) formation by VisA/B/C proteins. (a) Virginiamycin $S$ is composed of the following seven amino acids: HPA, L-Thr, $\mathrm{D}-\alpha$-aminobutyric acid (D-amBu), L-Pro, $N$ methyl-L-phenylalanine ( $L-M e P h e), L-4-o x o p i-$ pecolic acid (L-4-oxo-Pip) and L-phenylglycine (L-pheGly). (b) L-Lysine is enzymically converted to adenyl-HPA by VisA and VisB, and to L-Pip by VisC, respectively, in a stepwise manner. gene for $\mathrm{VM}$ production) in the vicinity of the VM biosynthetic gene cluster (Pulsawat et al., 2007). Based on genetic information from pristinamycin I biosynthesis, it was deduced that VisE is responsible for incorporation of the two amino acids (L-threonine and $\mathrm{D}$-amBu), and that VisF is a large NRPS for condensation of the last four amino acids in the VS core structure: L-proline, $N$-methylL-phenylalanine, 4-oxo-L-pipecolic acid and L-pheGly. With respect to the supply of necessary nonproteinogenic amino acids, four VS biosynthetic genes ( $v i s A, v i s B, v i s C$ and $v i s D$ ) have been identified in the right-hand region of the virginiamycin biosynthetic gene cluster (Namwat et al., 2002). These four genes are required for creation and activation of the first amino acid moiety, 3-hydroxypicolinic acid, and the sixth amino acid moiety, 4-oxo-L-pipecolic acid, in the VS structure (Fig. 1b). However, other pathways to synthesize $\mathrm{D}-\mathrm{amBu}$ and L-pheGly are still needed, and remain to be elucidated. Although $p$-hydroxyphenylglycine and 3,5-dihydroxyphenylglycine are typical nonproteinogenic amino acids occasionally found in several natural products, including the family of glycopeptide antibiotics (Lamb et al., 2006), L-pheGly is only found in the type B compounds in the streptogramin family. Insights into the biosynthetic pathway of L-pheGly are important for future efforts in combinatorial biosynthetic manipulation. However, although the committed synthesis of hydroxyphenylglycines is usually achieved by specialized enzymes encoded by the clustered biosynthetic genes for hydroxyphenylglycine-containing natural products (Hubbard et al., 2000), and this synthetic mechanism has been well studied, very little information is available for the biosynthetic gene(s) for L-pheGly.

Here, we provide genetic and chemical data to characterize the VS biosynthetic genes that are located in a boundary region of the supercluster, and demonstrate that $v i s G$ plays an important role in the biosynthesis of L-pheGly, a nonproteinogenic amino acid, in VS production.

\section{METHODS}

Bacterial strains, plasmids, media and growth conditions. Streptomyces virginiae strain MAFF 10-06014 from the National Food Research Institute (Tsukuba, Japan) was grown at $28{ }^{\circ} \mathrm{C}$ on ISP medium 2 (Becton, Dickinson and Company). Mannitol soya flour (MS) medium as described by Kieser et al. (2000) but supplemented with $10 \mathrm{mM} \mathrm{MgCl}_{2}$ was used for intergenic conjugation. For virginiamycin production, liquid f-medium was used as described previously (Nihira et al., 1988). Escherichia coli DH5 $\alpha$ was used for general DNA manipulation (Kieser et al., 2000; Sambrook \& Russell, 2001) and E. coli ET12567 (dam-13::Tn9 dcm-6 hsdM hsdS) containing the RP4 derivative pUZ8002 (Paget et al., 1999) was used for E. coli/Streptomyces conjugation. The plasmids used were pBluescript II SK for general cloning, pKC1132 (Bierman et al., 1992) for gene disruption and pLT101, a derivative of pSET152 (Bierman et al., 1992) containing the strong and constitutive promoter $e r m E p^{*}$ and a $t f d$ terminator, for gene complementation. All the primers are listed in Supplementary Table S1, available with the online version of this paper.

Transcriptional analysis by quantitative RT-PCR. Total RNAs were extracted from mycelia harvested by using an RNeasy Mini kit (Qiagen) and treated with DNase I (Takara Bio). The cDNA was synthesized using SuperScript III RNase $\mathrm{H}^{-}$Reverse Transcriptase (Invitrogen) and Random Primers (Invitrogen) according to the manufacturer's instructions. Quantitative RT-PCR (qRT-PCR) was performed using the Applied Biosystems 7300 Real-Time PCR System and SYBR Green PCR Master Mix (Applied Biosystems) according to the supplier's recommendations. The reaction conditions were as follows: $95{ }^{\circ} \mathrm{C}$ for $10 \mathrm{~min}$ followed by 40 cycles consisting of $15 \mathrm{~s}$ at $95{ }^{\circ} \mathrm{C}$ for denaturation and $1 \mathrm{~min}$ at $68{ }^{\circ} \mathrm{C}$ for annealing and extension. A final dissociation stage was performed to generate a melting curve and consequently verify the specificity of the amplification products. $r$ plA, encoding $50 \mathrm{~S}$ ribosomal protein $\mathrm{L} 1$ in S. virginiae (Katayama et al., 1996), was used as a negative control. Gene expression was measured in triplicate and normalized to the mRNA level of the $h r d B$-like gene [ $h r d B$ encodes the major sigma factor in Streptomyces coelicolor A3(2) (Buttner et al., 1990)] using the relative standard curve method, as the gene is expressed fairly constantly throughout growth.

Disruption of visG. A $2.3 \mathrm{~kb}$ visG-upstream fragment was amplified using the primer pair visG-AX/visG-AS, and digested with $\mathrm{XbaI}$ and 
SpeI. Similarly, a $2.3 \mathrm{~kb}$ visG-downstream fragment was amplified using the primer pair visG-BS/visG-BH, and digested with SpeI and HindIII. PCR was performed using high-fidelity PrimeSTAR HS DNA polymerase (Takara Bio) according to the manufacturer's recommendations. The fidelity of the amplified regions was confirmed by sequencing. The two resulting fragments were cloned together into the XbaI and HindIII sites of pBluescript II SK, and were recovered as a $4.5 \mathrm{~kb} \mathrm{XbaI/HindIII} \mathrm{fragment,} \mathrm{which} \mathrm{was} \mathrm{then} \mathrm{inserted} \mathrm{into} \mathrm{the}$ $X b a \mathrm{I}$ and HindIII sites of pKC1132 to generate pLT208 for visG disruption. E. coli ET12567(pUZ8002) harbouring pLT208 was conjugated with $S$. virginiae, and the wild-type gene was replaced with the disrupted allele $(\Delta v i s G)$ by homologous recombination. The genotype of the $\Delta v i s G$ disrupted candidates was confirmed by PCR analysis, and the visG disruptant was designated S. virginiae strain IC109.

Genetic complementation of the visG disruptant. The visG ORFs starting from different initiation codons were amplified by the primer pairs visGS-N/visG-C and visGL-N/visG-C, respectively, and each fragment was inserted into the EcoRV site of pBluescript II SK. The resulting plasmids were digested with $B a m \mathrm{HI}$, and were then cloned into the BamHI site of pLT101 (Pulsawat et al., 2009) to place the $v i s G$ ORF under the control of ermEp ${ }^{*}$, resulting in pLT209 and pLT210, respectively. By intergenic conjugation and integration, each plasmid was introduced into S. virginiae strain IC109, creating S. virginiae strains IC110 and IC111, respectively. The correct integration in the exconjugants was confirmed by PCR analysis.

Analysis of virginiamycin production. Virginiamycin in the culture broth was detected by a bioassay against Bacillus subtilis PCI219 (Yanagimoto, 1983) and reversed-phase $\mathrm{C}_{18}$-HPLC analysis as described by Pulsawat et al. (2007). Purified VS and VM were used as standards for HPLC analysis.

Feeding of the visG mutant with L-pheGly and L-pheGly analogues. S. virginiae strain IC109 was grown in $70 \mathrm{ml}$-medium as described previously. After incubation for $12 \mathrm{~h}$, L-pheGly (Tokyo Chemical Industry), D-(-)-2-(p-hydroxyphenyl)glycine (Wako Pure Chemical Industries), L-phenylalanine (Wako Pure Chemical Industries) or L-3,4-dihydroxyphenylalanine (Wako Pure Chemical Industries) was added to the culture. After incubation for $24 \mathrm{~h}$, the culture supernatants were analysed by a bioassay and reversed-phase HPLC.

\section{RESULTS}

\section{Cloning and in silico analysis of the region downstream of visE in the virginiamycin biosynthetic gene cluster}

Previously, we demonstrated that two genes are present at the left-hand extremity of the virginiamycin biosynthetic cluster: visE, which encodes an NRPS containing two A domains for incorporation of $\mathrm{L}-\mathrm{Thr}$ and $\mathrm{D}$-amBu into the VS structure, and an incomplete ORF $(v i s F)$, which partially encodes a protein containing one condensation (C) domain (Pulsawat et al., 2007). To isolate the complete vis $F$ gene and search for additional genes involved in virginiamycin biosynthesis, a cosmid library of the $S$. virginiae genome was screened by PCR. Using a partial nucleotide sequence of the visF gene, we identified one cosmid (2B9), the insert of which covered a $28 \mathrm{~kb}$ region downstream of visE and contained a complete ORF of visF and seven more ORFs based on an analysis with FramePlot 4.0beta (http://nocardia.nih.go.jp/fp4/) (Fig. 2a). The plausible function for each ORF was assigned by comparing the translated product with known proteins in public databases (Table 1).

VisF possesses NRPS modules including a total of four adenylation (A) domains, four peptidyl carrier protein (PCP) domains, four condensation (C) domains, one methylation (M) domain and one thioesterase (TE) domain. Bioinformatic analysis to predict the substrate

(a)

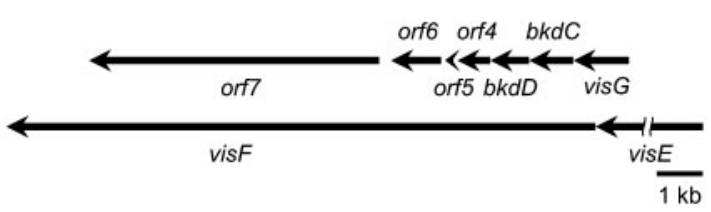

(b)

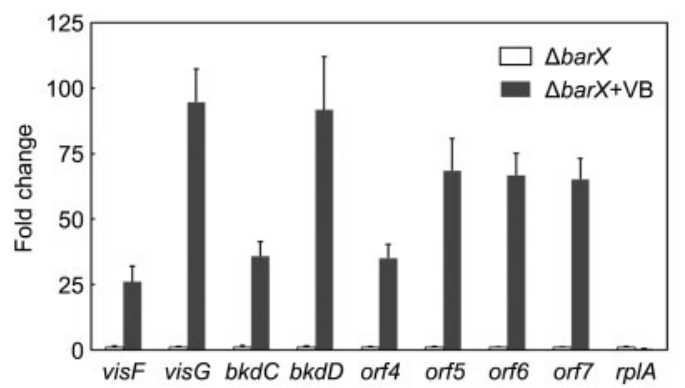

(c)

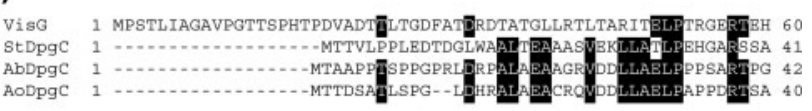

(d)

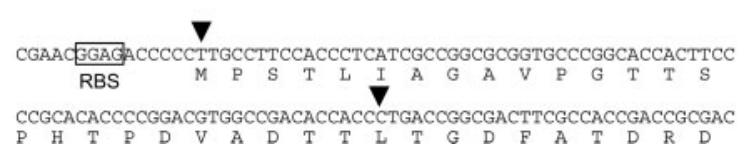

Fig. 2. (a) Gene organization of the sequenced $28 \mathrm{~kb}$ DNA region in the visE-flanking region, (b) transcriptional analysis of the identified genes by qRT-PCR, (c) amino acid sequence alignment of the N-terminal region of Vis $\mathrm{G}$ with those of other actinomycetes' $\mathrm{DpgC}$, and (d) the nucleotide sequence encoding the $\mathrm{N}$-terminus of VisG and the visG-upstream region. (a) The direction of visG transcription is the same as those of the visE and $v m s T$ genes. (b) Total RNA was extracted from mycelia harvested after incubation for $10 \mathrm{~h}$. VB-C $6\left(60 \mathrm{ng} \mathrm{ml}^{-1}\right)$ was added into the culture of the bar $X$ mutant after incubation for $8 \mathrm{~h}$. Virginiamycin production was observed after incubation for $10 \mathrm{~h}$ with exogenous VB addition. Fold change is relative to the expression of each gene without VB addition after incubation for $10 \mathrm{~h}$. Error bars represent standard deviations from triplicate experiments. (c) The numbers indicate the amino acid positions within each sequence. Black boxes in the alignment indicate positions at which the same amino acid is found in at least three of the four sequences: StDpgC (AAM80546) from S. toyocaensis, AbDpgC (CAC48380) from A. balhimycina and AoDpgC (CAA11787) from Amycolatopsis orientalis. (d) The putative ribosome-binding site (RBS) is shown in a box. The black triangles together with either TTG or CTG indicate the positions of probable initiation codons for the longer and shorter VisG protein, respectively. 
Table 1. Summary of ORFs in the cloned DNA

\begin{tabular}{|c|c|c|c|c|c|c|}
\hline Protein & Location & $\begin{array}{c}\text { Amino } \\
\text { acids }\end{array}$ & Proposed function & $\begin{array}{l}\text { Sequence similarity } \\
\text { (protein, origin) }\end{array}$ & $\begin{array}{c}\text { Identity/ } \\
\text { Similarity (\%) }\end{array}$ & Accession no. \\
\hline VisF & $1-14763$ & 4920 & NRPS for VS & SnbDE, S. pristinaespiralis & $67 / 92$ & СВH31051 \\
\hline VisG & $14796-16190$ & 464 & $\begin{array}{l}\text { Phenylacetyl-CoA } \\
\text { dioxygenase }\end{array}$ & DpgC, S. toyocaensis & $48 / 83$ & AAM80546 \\
\hline BkdC & $16187-17290$ & 367 & BCDH E1 $\alpha$ subunit & BkdA2, S. coelicolor A3(2) & $54 / 83$ & CAD55333 \\
\hline BkdD & $17290-18276$ & 328 & $\mathrm{BCDH}$ E1 $\beta$ subunit & BkdB2, S. coelicolor A3(2) & $74 / 93$ & CAB46953 \\
\hline ORF4 & 18273-19100 & 275 & Type II thioesterase & Ken1, Streptomyces violaceoruber & $41 / 77$ & CAQ52621 \\
\hline ORF5 & 19184-19402 & 72 & MbtH-like protein & Orf46, Streptomyces fungicidicus & $66 / 87$ & ABD65966 \\
\hline ORF6 & 19489-20754 & 421 & $\begin{array}{l}\text { Phenylglycine } \\
\text { aminotransferase }\end{array}$ & PgaT, A. balhimycina & $56 / 88$ & CAC48367 \\
\hline ORF7 & $21052-28320$ & 2422 & NRPS & SnaD, S. pristinaespiralis & $69 / 92$ & CBW45640 \\
\hline
\end{tabular}

specificity of the four A domains using NRPSpredictor (http://ab.inf.uni-tuebingen.de/software/NRPSpredictor/ welcome.html) revealed that the first $\mathrm{A}$ domain $\left[\mathrm{VisFA}_{1}\right.$, DVQYAAHVMK is the 10 amino acid code of the substrate recognition sequence as defined by Stachelhaus et al. (1999)] is specific for the incorporation of proline, the second $\mathrm{A}$ domain (VisFA 2 , DAWTVAAVCK) recognizes phenylalanine as a substrate, and the third and fourth $\mathrm{A}$ domains (VisFA 3 , DFQFFGVAVK; VisFA 4 , DIYLWGLLGK) specifically incorporate pipecolic acid and L-pheGly, respectively. The $\mathrm{M}$ domain followed the $\mathrm{VisFA}_{2}$ domain, presumably giving methylphenylalanine as the fourth amino acid of the VS structure. In addition, VisF showed high similarity with SnbDE, a pristinamycin I synthetase from S. pristinaespiralis (de Crécy-Lagard et al., 1997), suggesting that VisF probably assembles the fourth to seventh amino acids (L-proline, methyl-L-phenylalanine, L-pipecolic acid and L-pheGly) into the VS core structure. The visF-downstream gene visG encodes a protein with homology to a hydroxyphenylacetylCoA dioxygenase, such as DpgC of Streptomyces toyocaensis and Amycolatopsis balhimycina, which are necessary for dihydroxyphenylglycine biosynthesis (Pfeifer et al., 2001; Pootoolal et al., 2002). The next two genes, $b k d C$ and $b k d D$, encode $\mathrm{E} 1 \alpha$ and $\mathrm{E} 1 \beta$ subunits of the branched-chain $\alpha$-keto acid dehydrogenase $(\mathrm{BCDH})$ complex that is responsible for oxidative decarboxylation (Skinner et al., 1995). The following gene, orf4, encodes a type II thioesterase. Type II thioesterases are present in many biosynthetic gene clusters for nonribosomal peptides, where they have corrective functions by hydrolysing aberrant substrates from the respective PCP domains (Heathcote et al., 2001). The gene product of orf5 exhibits similarity to the MbtH-like protein. $m b t H$-like genes are frequently found in the biosynthetic gene clusters of nonribosomal peptides and siderophores, and have recently been reported to be necessary for modulation of the enzymic activity of NRPS (Zhang et al., 2010). The protein encoded by the next gene, orf6, has homology to aminotransferases involved in di- or monohydroxyphenylglycine biosynthesis, such as PgaT of $A$. balhimycina (Pfeifer et al., 2001). The last gene, orf7, encodes an NRPS with homology to $\mathrm{SnaD}$, a pristinamycin II synthetase, which introduces the final residue proline into pristinamycin II (Blanc et al., 1994). ORF7 possesses NRPS modules including a total of two C domains, two PCP domains, one TE domain, one A domain, and one other domain (TIGR01720) that is positioned between amino acids 1414 and 1572 and has an unknown function. However, it seems that the organization of the modules and domains in the ORF7 protein does not follow the NRPS collinearity principle, and the sole A domain $\left(\mathrm{ORF}_{1} \mathrm{~A}_{1}\right.$, DVHHVTAYSK) shows no homology to the known A domain. From these analyses, it is difficult to predict the involvement of the ORF7 protein in VM biosynthesis at present.

\section{Transcriptional control by virginiae butanolide of the newly identified genes}

Virginiamycin production in S. virginiae is controlled by a $\gamma$-butyrolactone autoregulator, virginiae butanolide (VB), and its cognate receptor BarA as a DNA-binding transcriptional repressor (Kinoshita et al., 1997). The VB biosynthetic pathway requires enzymic function of BarX, an AfsA-family protein (Lee et al., 2010). To investigate the effect of VB on transcription of the newly identified genes, the transcriptional levels were examined by qRT-PCR analysis (Fig. 2b). The barX mutant has no ability to produce virginiamycin. On the other hand, when VB is added exogenously to the culture of the barX mutant at $8 \mathrm{~h}$, virginiamycin production occurs from $10 \mathrm{~h}$ (Lee et al., 2010). The transcription levels of the identified genes showed a significant relative increase of 26- to 96-fold in the presence of external VB compared to those without VB addition. These results indicated that the eight identified genes downstream of visE are new members of the VB regulon, in addition to the virginiamycin biosynthetic genes.

Within the VB-responsive genes, the mRNA level of vis $G$ is highly dependent on VB (about 96-fold). Accordingly, the transcriptional level of visG was compared between the wild-type strain and the $\operatorname{barB}$ disruptant, which shows earlier and higher-level production of virginiamycins. $\mathrm{BarB}$, whose expression is under the positive control of 
$\mathrm{VB}$ via BarA, is a transcriptional repressor in the early process of virginiamycin biosynthesis (Matsuno et al., 2004). The results showed that after incubation for $10 \mathrm{~h}$ vis $G$ transcription increased up to $270( \pm 41.5)$-fold in the $\operatorname{bar} B$ disruptant compared with the wild-type strain (average of three independent experiments). Taken together with the homology analysis described above, these results supported the idea that VisG is one of the virginiamycin biosynthetic enzymes and supplies L-pheGly.

\section{The visG disruptant lacks the ability to produce VS, not VM}

To provide direct evidence that $v i s G$ is involved in virginiamycin production, we disrupted vis $G$ by in-frame deletion of 353 amino acids. The resulting mutants were analysed by PCR in order to detect the size of the replaced allelic visG gene (Fig. 3a). The mutant IC109 grew normally in liquid cultivation and showed morphological characteristics on solid medium identical to those of the wild-type strain (results not shown). These results indicated that vis $G$ is not involved in either primary metabolism or morphological differentiation. To assess virginiamycin production, the visG disruptant was cultivated in liquid medium and the culture supernatant was analysed by a bioassay against B. subtilis (Fig. 3b). The results showed that the visG disruptant (strain IC109) exhibited a smaller clear zone than that of the wild-type strain. Because this phenomenon could have been due either to a compositional or quantity change of virginiamycin, the culture supernatant was analysed by HPLC. As shown in Fig. 3(c), a peak corresponding to VS (25.7 $\mathrm{min})$ was missing in strain IC109, whereas the peak corresponding to VM remained intact. The peak at 25.9 min was eluted later than VS and thus was not VS. Thus, inactivation of visG completely abolished VS production, but not VM production, indicating that VisG is necessary only for VS biosynthesis.

To ensure that inactivation of vis $G$ was the sole reason for the observed loss of the VS production, the intact vis $G$ gene was reintroduced into the mutant strain. FramePlot analysis and sequence alignment analysis with DpgC proteins predicted two plausible initiation codons for the VisG protein (Fig. 2c, d): one is a TTG codon with a putative ribosome-binding site (RBS) located 7 bp upstream and the other is a CTG codon with no distinct RBS. Furthermore, because there is only a narrow intergenic region (about $40 \mathrm{bp}$ ) between $v i s F$ and $v i s G$, vis $G$ may form a bicistronic operon with visF, with no definite promoterlike sequences in the region immediately upstream of visG. Thus, we constructed plasmids that each contained different vis $G$ genes driven by the strong constitutive ermEp $p^{*}$ promoter. Introduction of pLT209, containing a shorter visG gene, into the mutant IC109 did not restore VS production (results not shown). On the other hand, complementation of the mutant IC109 with pLT210, containing a longer vis $G$ gene, restored the VS production to a level similar to that of the wild-type strain (Fig. 3b, c). (a)

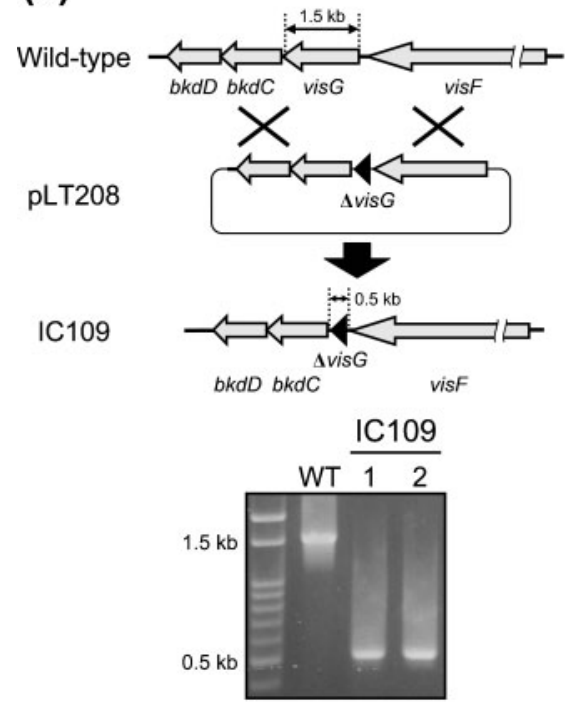

(b)

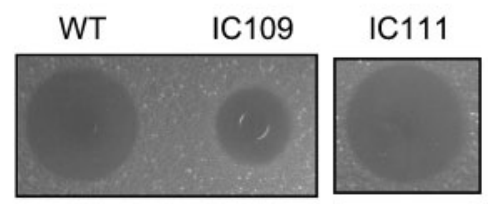

(c)

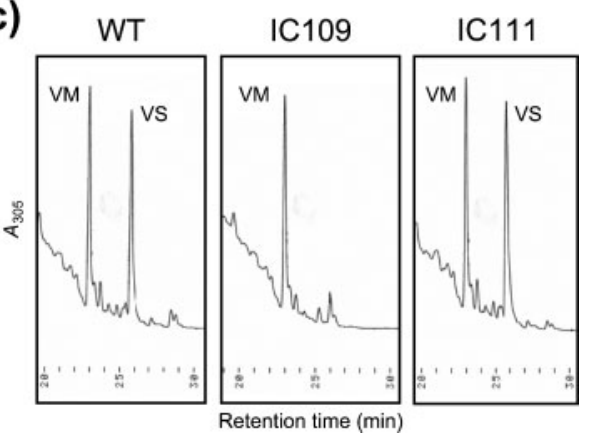

Fig. 3. Inactivation of visG by gene replacement (a), and bioassay (b) and HPLC analysis (c) of virginiamycin production. (a) Schematic representation of visG disruption. The black triangles indicate the deleted vis $G$ gene $(\Delta v i s G)$. PCR analysis of chromosomal DNA from the wild-type strain (WT) and $\Delta$ visG disruptants (IC109-1 and IC109-2) using the primer pair visG$\mathrm{DF} / \mathrm{visG}-\mathrm{DR}$ for detection of the $\Delta$ visG mutation. (b) Bioassay of $24 \mathrm{~h}$ culture broths against $B$. subtilis. S. virginiae strain IC111 is a visG-complemented visG disruptant in which pLT210 is integrated into the chromosome. (c) HPLC analysis of virginiamycin production. Production profiles corresponding to the retention time (19-30 min) with detection at $305 \mathrm{~nm}$ are shown. The peaks of virginiamycin $M_{1}(V M)$ and virginiamycin $S(V S)$ are indicated. 
These results demonstrated that functional visG should start from the TTG codon to encode a 464 amino acid protein and should be essential for the VS biosynthesis.

\section{Addition of L-pheGly restores the deficiency of VS production}

Due to the similarity of VisG to a hydroxyphenylacetylCoA dioxygenase, VisG is predicted to be involved in the biosynthesis of L-pheGly. To confirm this possibility, LpheGly (Fig. 4a) was added to the production medium of the vis $G$ disruptant and the biological activity of the culture supernatant was assessed. Bioassay analysis (Fig. 4b) as well as HPLC analysis (Fig. 4c) demonstrated that the addition of L-pheGly resulted in recovery of the VS production in the vis $G$ disruptant, indicating that the loss of VS production was due to a lack of L-pheGly and implying that VisG would be an L-pheGly-producing enzyme in the L-pheGly biosynthetic pathway.

In contrast to the case of L-pheGly addition, addition of LpheGly analogues, such as p-hydroxyphenylglycine (Fig. 4a), L-phenylalanine, or L-3,4-dihydroxyphenylalanine (LDOPA), did not result in any difference in the size of the clear zone (Fig. 4b) or in the HPLC profiles (Fig. 4c), suggesting that these analogues are not incorporated into the elongating VS backbone structure, probably due to the strict recognition of the L-pheGly structure by the enzyme responsible, VisF.

\section{DISCUSSION}

Hydroxylated phenylglycines are found in various natural products of peptidic nature, such as glycopeptide antibiotics (vancomycin and teicoplanin) and other antimicrobial compounds (ramoplanin and calcium-dependent antibiotic), and are known, as in the case of p-hydroxyphenylglycine and 3,5-dihydroxyphenylglycine, to be essential for rigidifying side-chain cross-links of the peptide scaffold. Although the biosynthetic pathways of p-hydroxyphenylglycine and 3,5dihydroxyphenylglycine have been well studied (Hubbard et al., 2000; Tseng et al., 2004), there is little available information on genes or pathways for the biosynthesis of a similar nonproteinogenic amino acid, L-pheGly, that is only found in streptogramin antibiotics. In the present study, we verified that VisG is an L-pheGly provider that is essential for the biosynthesis of VS, a streptogramin type B antibiotic. VisG resembles the 3,5-dihydroxyphenylacetyl (DPA)-CoA 1,2-dioxygenase $\mathrm{DpgC}$, which is reported to incorporate one oxygen atom of $\mathrm{O}_{2}$ at C-2 and another at C-1 of DPA-CoA with cleavage of the thioester bond to form dihydroxyphenyglyoxylate (Tseng et al., 2004). With respect to L-pheGly biosynthesis, we propose that phenylacetyl-CoA would be converted to benzoylformate by VisG, although in vitro experiments will be needed to clarify the detailed reaction mechanism. Following the VisG reaction, an aminotransferase would convert the benzoylformate to L-pheGly, which (a)

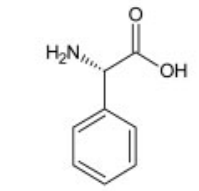

L-Phenylglycine (L-pheGly)

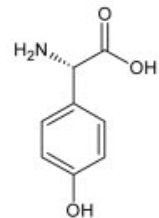

$p$-Hydroxyphenylglycine (Hpg)

(b)
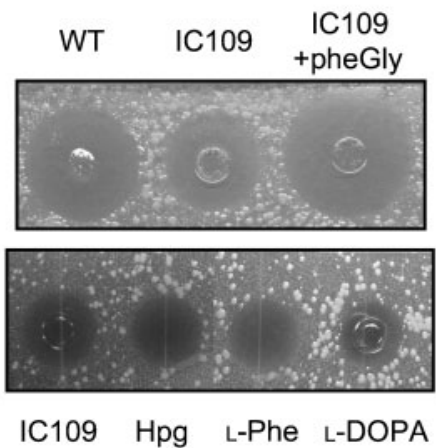

(c)

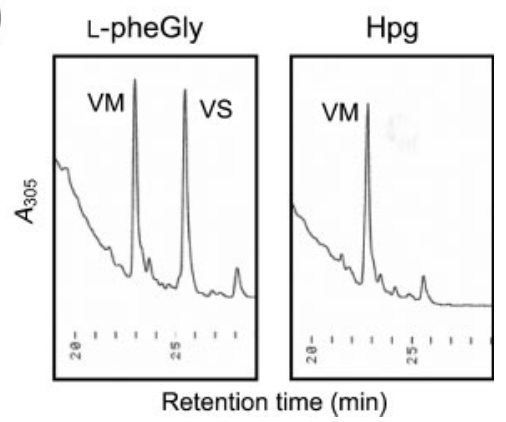

Fig. 4. External addition of L-pheGly for chemical complementation, and L-pheGly analogues for biosynthesis of the VS derivative. (a) Chemical structure of L-pheGly and Hpg. (b) L-pheGly was added to a final concentration of $100 \mu \mathrm{M}$ after incubation for $12 \mathrm{~h}$ (upper panel), and the L-pheGly analogues Hpg, L-phenylalanine (L-Phe) and L-3,4-dihydroxyphenylalanine (L-DOPA) were added individually to a final concentration of $500 \mu \mathrm{M}$ after incubation for $12 \mathrm{~h}$ (lower panel). (c) Production profiles corresponding to the retention time (19-29 $\mathrm{min}$ ) with detection at $305 \mathrm{~nm}$ are shown. The peaks of VM and VS are indicated.

would eventually be activated and incorporated into VS by the action of VisF.

Four genes ( $v i s G, b k d C, b k d D$ and orf4) have start and stop codons which overlap each other sequentially and their transcription was induced by the external addition of VB, suggesting that vis $G$ might form a tetracistronic operon with $b k d C, b k d D$ and orf4. Increased transcription of visF with the addition of $\mathrm{VB}$ was also observed, and the intergenic region between visF and vis $G$ is likely to be short, implying that visG would be expressed polycistronically with visF, $b k d C, \quad b k d D$ and orf4. In the course of 
constructing the visG-disruption plasmid, one of the plasmids was unintentionally made to contain a $34 \mathrm{bp}$ deletion in the coding region of $b k d D$, which presumably encodes the $\mathrm{E} 1 \beta$ subunit of the $\mathrm{BCDH}$ complex. Introduction of this plasmid resulted in double mutations $(\Delta v i s G \Delta b k d D)$. The strain harbouring the double mutations showed no VS production, similar to that of the visG disruptant (results not shown). However, complementation by intact visG completely restored VS production to a level similar to that of the wild-type strain without any change of VM production, indicating that $b k d D$ (presumably as well as $b k d C$ encoding the cognate E1 $\alpha$ subunit) at least is not involved in the biosynthesis of virginiamycins. Although phenotypic analysis of the orf4 mutant is under way to further clarify the borders of the virginiamycin cluster, at present, vis $G$ is the VS biosynthetic gene in the most left-hand extremity within the clustered virginiamycin biosynthetic genes.

Expanding the diversity of the virginiamycin scaffold could facilitate chemical modification, affecting the water solubility, size and receptor affinity of the molecules. With respect to alteration of non-ribosomal peptide scaffolds, $p$ hydroxyphenylglycine (Hpg) or L-3,5-dihydroxyphenylglycine (DPG) residues are attractive targets for chemical modification, because the hydroxyl groups on the aromatic ring are easily modified by sulfation (Lamb et al., 2006), and chlorine atoms can be introduced into the DPG residue by a halogenase (Puk et al., 2002). In the present study, we attempted to make VS derivatives by feeding the vis $G$ disruptant with L-pheGly analogues harbouring a mono- or dihydroxyl group on the aromatic ring. However, the incorporation of L-pheGly analogues seems to be hampered by strict substrate recognition of the A domain, $\mathrm{VisFA}_{4}$, of the corresponding NRPS. The Hpgincorporating A domains show many variants of the substrate recognition sequence, such as BpsB-m1 and BpsB-m2 for balhimycin biosynthesis (DIFHLGLLCK and DAVHLGLLCK, respectively), and EndC-m2 for enduracidin biosynthesis (DAYHLGMLCK) (Recktenwald et al., 2002; Yin \& Zabriskie, 2006). Mutagenesis and replacement analyses of the L-pheGly-incorporating A domain $\left(\mathrm{VisFA}_{4}\right)$ are currently under way in our laboratory. Together with the biochemical analysis, these studies will provide useful information for forced incorporation of Hpg and DPG into the VS core structure.

\section{ACKNOWLEDGEMENTS}

This work is a part of the $\mathrm{PhD}$ dissertation of F. N. We thank Satoshi Harashima, Minetaka Sugiyama and Kiyoko Miyamoto for assistance with qRT-PCR analysis. This work was supported in part by a grant for the Joint Program in the Field of Biotechnology under the Japan Society for Promotion of Science (JSPS), the National Research Council of Thailand and the National Science and Technology Development Agency of Thailand to T.N., by a Grant-in-Aid for Scientific Research (B) (no. 21360404) from JSPS to T.N. and S. K., and by a scholarship from the Ministry of Education, Culture, Sports, Science and Technology of Japan to F. N.

\section{REFERENCES}

Barrière, J. C., Berthaud, N., Beyer, D., Dutka-Malen, S., Paris, J. M. \& Desnottes, J. F. (1998). Recent developments in streptogramin research. Curr Pharm Des 4, 155-180.

Bierman, M., Logan, R., O'Brien, K., Seno, E. T., Rao, R. N. \& Schoner, B. E. (1992). Plasmid cloning vectors for the conjugal transfer of DNA from Escherichia coli to Streptomyces spp. Gene 116, 43-49.

Blanc, V., Blanche, F., Crouzet, J., Jacques, N., Lacroix, P., Thibaut, D., Zagorec, M., Debussche, L. \& de Crécy-Lagard, V. (1994). Polypeptides involved in streptogramin biosynthesis, nucleotide sequences coding for said polypeptides and use thereof. US Patent no. 5891695 .

Buttner, M. J., Chater, K. F. \& Bibb, M. J. (1990). Cloning, disruption, and transcriptional analysis of three RNA polymerase sigma factor genes of Streptomyces coelicolor A3(2). J Bacteriol 172, 3367-3378.

de Crécy-Lagard, V., Saurin, W., Thibaut, D., Gil, P., Naudin, L., Crouzet, J. \& Blanc, V. (1997). Streptogramin B biosynthesis in Streptomyces pristinaespiralis and Streptomyces virginiae: molecular characterization of the last structural peptide synthetase gene. Antimicrob Agents Chemother 41, 1904-1909.

Di Giambattista, M., Chinali, G. \& Cocito, C. (1989). The molecular basis of the inhibitory activities of type A and type B synergimycins and related antibiotics on ribosomes. J Antimicrob Chemother 24, 485-507.

Heathcote, M. L., Staunton, J. \& Leadlay, P. F. (2001). Role of type II thioesterases: evidence for removal of short acyl chains produced by aberrant decarboxylation of chain extender units. Chem Biol 8, 207220.

Hubbard, B. K., Thomas, M. G. \& Walsh, C. T. (2000). Biosynthesis of L- $p$-hydroxyphenylglycine, a non-proteinogenic amino acid constituent of peptide antibiotics. Chem Biol 7, 931-942.

Katayama, M., Sakai, Y., Okamoto, S., Ihara, F., Nihira, T. \& Yamada, Y. (1996). Gene organization in the $a d a-r p l L$ region of Streptomyces virginiae. Gene 171, 135-136.

Kieser, T., Bibb, M. J., Buttner, M. J., Chater, K. F. \& Hopwood, D. A. (2000). Practical Streptomyces Genetics. Norwich, UK: John Innes Foundation.

Kinoshita, H., Ipposhi, H., Okamoto, S., Nakano, H., Nihira, T. \& Yamada, Y. (1997). Butyrolactone autoregulator receptor protein (BarA) as a transcriptional regulator in Streptomyces virginiae. J Bacteriol 179, 6986-6993.

Lamb, S. S., Patel, T., Koteva, K. P. \& Wright, G. D. (2006). Biosynthesis of sulfated glycopeptide antibiotics by using the sulfotransferase StaL. Chem Biol 13, 171-181.

Lee, Y. J., Kitani, S. \& Nihira, T. (2010). Null mutation analysis of an $a f_{s} A$-family gene, $b a r X$, that is involved in biosynthesis of the $\gamma$ butyrolactone autoregulator in Streptomyces virginiae. Microbiology 156, 206-210.

Manzella, J. P. (2001). Quinupristin-dalfopristin: a new antibiotic for severe Gram-positive infections. Am Fam Physician 64, 18631866.

Matsuno, K., Yamada, Y., Lee, C. K. \& Nihira, T. (2004). Identification by gene deletion analysis of $b a r B$ as a negative regulator controlling an early process of virginiamycin biosynthesis in Streptomyces virginiae. Arch Microbiol 181, 52-59.

Namwat, W., Kamioka, Y., Kinoshita, H., Yamada, Y. \& Nihira, T. (2002). Characterization of virginiamycin $S$ biosynthetic genes from Streptomyces virginiae. Gene 286, 283-290.

Nihira, T., Shimizu, Y., Kim, H. S. \& Yamada, Y. (1988). Structureactivity relationships of virginiae butanolide $C$, an inducer of 
virginiamycin production in Streptomyces virginiae. J Antibiot (Tokyo) 41, 1828-1837.

Paget, M. S., Chamberlin, L., Atrih, A., Foster, S. J. \& Buttner, M. J. (1999). Evidence that the extracytoplasmic function sigma factor $\sigma^{\mathrm{E}}$ is required for normal cell wall structure in Streptomyces coelicolor A3(2). J Bacteriol 181, 204-211.

Pfeifer, V., Nicholson, G. J., Ries, J., Recktenwald, J., Schefer, A. B., Shawky, R. M., Schröder, J., Wohlleben, W. \& Pelzer, S. (2001). A polyketide synthase in glycopeptide biosynthesis: the biosynthesis of the non-proteinogenic amino acid (S)-3,5-dihydroxyphenylglycine. J Biol Chem 276, 38370-38377.

Pootoolal, J., Thomas, M. G., Marshall, C. G., Neu, J. M., Hubbard, B. K., Walsh, C. T. \& Wright, G. D. (2002). Assembling the glycopeptide antibiotic scaffold: the biosynthesis of A47934 from Streptomyces toyocaensis NRRL15009. Proc Natl Acad Sci U S A 99, 8962-8967.

Puk, O., Huber, P., Bischoff, D., Recktenwald, J., Jung, G., Süssmuth, R. D., van Pée, K. H., Wohlleben, W. \& Pelzer, S. (2002). Glycopeptide biosynthesis in Amycolatopsis mediterranei DSM5908: function of a halogenase and a haloperoxidase/perhydrolase. Chem Biol 9, 225-235.

Pulsawat, N., Kitani, S. \& Nihira, T. (2007). Characterization of biosynthetic gene cluster for the production of virginiamycin $\mathrm{M}$, a streptogramin type A antibiotic, in Streptomyces virginiae. Gene 393, $31-42$.

Pulsawat, N., Kitani, S., Fukushima, E. \& Nihira, T. (2009). Hierarchical control of virginiamycin production in Streptomyces virginiae by three pathway-specific regulators: VmsS, VmsT and VmsR. Microbiology 155, 1250-1259.

Recktenwald, J., Shawky, R., Puk, O., Pfennig, F., Keller, U., Wohlleben, W. \& Pelzer, S. (2002). Nonribosomal biosynthesis of vancomycin-type antibiotics: a heptapeptide backbone and eight peptide synthetase modules. Microbiology 148, 1105-1118.

Sambrook, J. \& Russell, D. W. (2001). Molecular Cloning: a Laboratory Manual, 3rd edn. Cold Spring Harbor, NY: Cold Spring Harbor Laboratory.

Skinner, D. D., Morgenstern, M. R., Fedechko, R. W. \& Denoya, C. D. (1995). Cloning and sequencing of a cluster of genes encoding branched-chain $\alpha$-keto acid dehydrogenase from Streptomyces avermitilis and the production of a functional E1 $[\alpha \beta]$ component in Escherichia coli. J Bacteriol 177, 183-190.

Stachelhaus, T., Mootz, H. D. \& Marahiel, M. A. (1999). The specificity-conferring code of adenylation domains in nonribosomal peptide synthetases. Chem Biol 6, 493-505.

Tseng, C. C., Vaillancourt, F. H., Bruner, S. D. \& Walsh, C. T. (2004). $\mathrm{DpgC}$ is a metal- and cofactor-free 3,5-dihydroxyphenylacetyl-CoA 1,2-dioxygenase in the vancomycin biosynthetic pathway. Chem Biol 11, 1195-1203.

Yamada, Y., Nihira, T. \& Sakuda, S. (1997). Butyrolactone autoregulators, inducers of virginiamycin in Streptomyces virginiae. In Biotechnology of Antibiotics, pp. 63-79. Edited by W. R. Strohl. New York: Marcel Dekker.

Yanagimoto, M. (1983). Novel actions of inducer in staphylomycin production by Streptomyces virginiae. J Ferment Technol 61, 443-448.

Yin, X. \& Zabriskie, T. M. (2006). The enduracidin biosynthetic gene cluster from Streptomyces fungicidicus. Microbiology 152, 2969-2983.

Zhang, W., Heemstra, J. R., Jr, Walsh, C. T. \& Imker, H. J. (2010). Activation of the pacidamycin PacL adenylation domain by $\mathrm{MbtH}-$ like proteins. Biochemistry 49, 9946-9947.

Edited by: J.-H. Roe 